\title{
REVIEW
}

\section{Breast Cancer Microenvironment Cross Talk through Extracellular Vesicle RNAs}

\author{
Samrita Dogra* and Bethany N. Hannafon ${ }^{* \dagger}$
}

From the Department of Obstetrics and Gynecology* and the Stephenson Cancer Center, ${ }^{\dagger}$ University of Oklahoma Health Sciences Center, Oklahoma City, Oklahoma

Accepted for publication March 31, 2021.

Address correspondence to Bethany N. Hannafon, Ph.D., Department of Obstetrics and Gynecology, Section of Gynecologic Oncology, University of Oklahoma Health Sciences Center, 975 NE 10th St., BRC 1274A, Oklahoma City, OK 73104. E-mail: bethany hannafon@ouhsc.edu.

\begin{abstract}
Exploration of extracellular communication has been at the forefront of research efforts in recent years. However, the mechanisms of cell-to-cell communication in complex tissues are poorly understood. What is clear is that cells do not exist in isolation, that they are constantly interacting and communicating with cells in the immediate vicinity and with cells at a distance. Intercellular communication by the release of small extracellular vesicles, called exosomes, loaded with RNAs is one mechanism by which cells communicate. In recent years, research has shown that exosomes, a class of extracellular vesicles, can play a major role in the pathogenesis of breast cancer. Specifically, exosomes have been demonstrated to play a role in promoting primary cancer development, invasion, metastasis, and chemotherapeutic resistance. This review summarizes what is known about the mechanisms of exosomemediated transfer of RNAs among cells in the breast microenvironment and discusses outstanding questions and the potential for new therapeutic intervention targeted at these interactions. (Am J Pathol 2021, 191: 1330-1341; https://doi.org/10.1016/j.ajpath.2021.03.014)
\end{abstract}

The mammary gland is a complex combination of multiple cell types that are distinctly organized into a glandular system and a surrounding supportive stroma that together generate a functioning organ. The mammary duct is composed of secretory luminal epithelial cells that line the internal face of the duct, surrounded by contractile myoepithelial cells and bounded by a basement membrane that maintains the epithelial cell polarity. The ducts are surrounded by an extracellular matrix (ECM) and various types of stromal cells, including fibroblasts, endothelial cells, immune cells, and adipocytes. Breast cancer development occurs through the accumulation of genetic and epigenetic events that lead to the proliferation of the luminal epithelial cells, changes in the basal membrane, loss of polarity, and a diminished myoepithelial cell layer, ultimately leading to the breakdown of the mammary ductal structure and breast cancer cell invasion. ${ }^{1}$ Whether this breakdown is directly due to the uncontrolled growth of the cancer cells or primarily due to extrinsic signaling events is presently unclear. Current research has shown that the intrinsic factors, such as genomic and transcriptomic changes, present in pre-invasive breast cancer are virtually indistinguishable from invasive breast cancer. $^{2-6}$ This suggests that extrinsic factors may play a predominant role in breast cancer progression and that breast cancer is not simply a disease of genetic and epigenetic events within the cell, but rather an ecological disease influenced by its physical surroundings. ${ }^{7,8}$ As such, a significant body of evidence has documented the importance of the cross talk between tumor cells and the surrounding stroma from cancer initiation to metastatic progression. ${ }^{9}$ Stromal cells, such as cancer-associated fibroblasts (CAFs), promote cancer growth by producing growth factors, proteases, and ECM proteins that recruit endothelial cells to generate new vasculature for increased nutrient uptake and immune effector proteins that generate

\footnotetext{
Supported by the Oklahoma Center for the Advancement of Science and Technology HR17-052 (B.N.H.).

Disclosures: None declared.

This article is a part of a review series on the role of the tumor microenvironment in breast cancer pathogenesis. B.N.H. did not serve as the Guest Editor on this manuscript.
} 
an anergic immune response, resulting in a permissive microenvironment that increases tumor growth and metastasis. Also, stromal adipocytes secrete adipokines and other chemokines and promote breast cancer cell proliferation, invasion, and metastatic progression. ${ }^{10}$ Although the cancer may originate within the epithelial cell layers of the mammary duct, it is the surrounding cells that provide the supportive environment for continued tumor growth. Therefore, a better understanding of the mechanisms of intercellular communication between the various cell populations within a breast tumor will provide a more complete view of the biology of breast tumor growth and reveal new methods for the treatment and prevention of breast cancer.

Exosomes are a class of endosome-derived nanometersized extracellular vesicles, also known as small extracellular vesicles, that mediate cell-to-cell communication by encapsulating lipids, proteins, and nucleic acids from the cell of origin and delivering them to a target cell via autocrine, paracrine, or endocrine signaling mechanisms. ${ }^{11}$ In recent years, exosomes have emerged as critical players in tumor progression with a described role in each progressive stage of tumor growth to metastatic dissemination. ${ }^{12}$ For example, extracellular vesicles from transformed cells enriched with specific cargo can transform normal cells and promote primary tumor growth in vivo. ${ }^{13}$ In addition, nonmalignant human breast cells have been shown to form tumors in mice when treated with cell line- or patientderived breast cancer exosomes. ${ }^{14}$ Exosomes secreted from cancer cells contribute to cancer progression by transferring oncogenic factors that modify the tumor microenvironment (TME). The release and uptake of tumorassociated exosomes promote a permissive TME that can support tumor growth, establish new vasculature, remodel the extracellular matrix, enable invasion and migration, and promote premetastatic niche formation, therapeutic resistance, and escape of tumor immune surveillance. ${ }^{11,12}$

The contents of exosomes are diverse, and their cargo reflects the complex and heterogeneous changes associated with growing tumors. Exosome cargo includes a variety of proteins, including oncoproteins, that are commonly amplified in cancer, that can accumulate in exosomes, and that on release regulate the TME and stromal response of tumors. DNA is also commonly identified in exosomes, and cancer-associated mutated DNA fragments have been identified. ${ }^{15}$ The RNA cargo of exosome is highly diverse, and several classes of RNAs have been detected, including protein-coding mRNAs and noncoding RNAs, such as rRNA, tRNA, miRNA, Piwi-interacting RNA, snRNA, small nucleolar RNA, long-noncoding RNA (lncRNA), and circular RNA. ${ }^{16,17}$ More importantly, the RNAs transferred by exosome are functional and can result in the translation of new proteins by transport of mRNAs ${ }^{18,19}$ or specific regulation of expression programs by transfer of miRNAs. ${ }^{20}$ The biogenesis, content loading of exosomes, and biological mechanisms associated with general exosome transfer have been reviewed elsewhere, ${ }^{21,22}$ and are not covered in this review. This review summarizes what is presently known about the role of exosome-related RNA cross talk in the breast cancer microenvironment (Figure 1). It also discusses outstanding questions and challenges in understanding the biological contribution of extracellular vesicle RNAs to breast cancer development and progression.

\section{Homotypic Interactions}

Breast cancers arise from the epithelial cell layers lining the mammary duct and lobules, where primary transformation occurs. ${ }^{1}$ In the pre-invasive stage of ductal breast cancer development, the transformed and proliferative ductal epithelial cells remain within the mammary ductal structure surrounded by an intact layer of myoepithelial cells and a basal lamina. These pre-invasive lesions, called ductal carcinoma in situ, are nonobligate precursors to invasive ductal carcinoma. One of the major gaps in exosome research is the current lack in understanding of the spatial distribution and temporal movement of exosomes within the developing breast cancer microenvironment. However, a few recent studies demonstrate the use of label-free optical contrast imaging to visualize exosomes within carcinogen-induced rat mammary tumors and ex vivo and intra-operative human breast tumors. $^{23-25}$ They show an increased density of exosomes near the tumor and a decreased density of exosomes further from the tumor, suggesting a diffusion-driven exosome distribution. The quantified exosome density also correlated with pathologic diagnoses, histologic grade of invasive ductal carcinoma, nuclear grade of ductal carcinoma in situ, and the tumor-to-margin distance. ${ }^{23}$ Interestingly, increased densities of exosomes were found inside desmoplastic regions, which are considered a histopathologic risk factor for breast cancer invasion, compared with the ECM of early-phase breast tumors, suggesting more interactive exosome communication at these sites. ${ }^{23}$ Progress has been made in understanding the dynamics and distribution of exosomes within tissues; meanwhile, a larger body of work has examined the mechanisms by which breast cancer-derived exosomes facilitate intercellular communication via the transfer of RNAs between breast cancer cells. The following sections focus on what is currently known about these homotypic exosome-associated RNA interactions.

\section{Oncogenic Transformation and Cancer Progression}

Breast cancer cells facilitate a tumor permissive microenvironment through the transfer of exosomes to normal human mammary epithelial cells (HMECs). One of the earliest studies to examine the role of exosome transfer to HMECs found that exosomes released by breast cancer cells could be taken up by HMECs and that this interaction induces reactive oxygen species production, autophagy, the DNA damage response, and activation and stabilization of p53. In a reciprocal relationship, the conditioned medium 


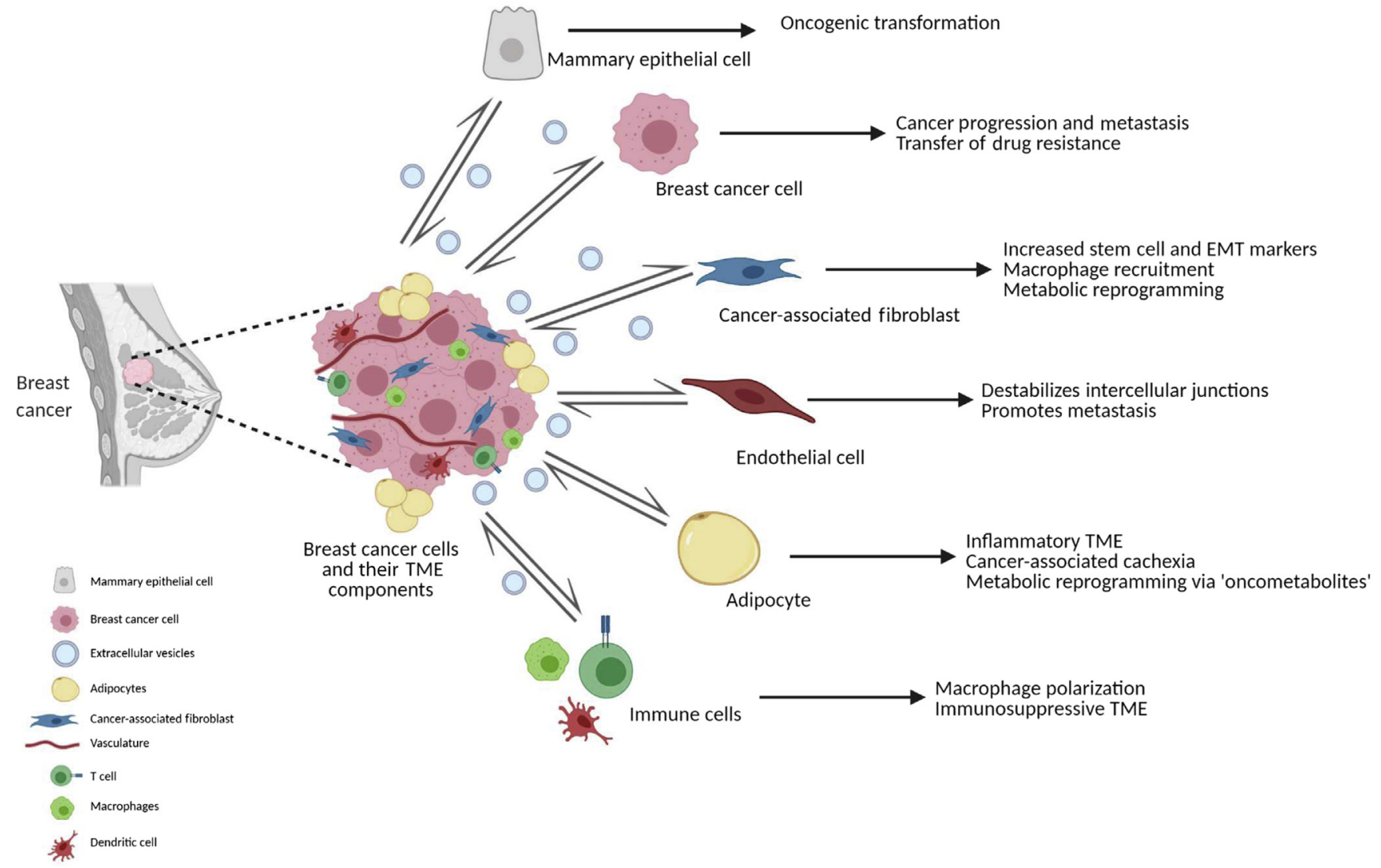

Figure 1 Schematic depiction of bidirectional communication between breast cancer cells and tumor microenvironment (TME), mediated by extracellular vesicles. The breast cancer TME is a heterogeneous mixture composed of adipocytes, fibroblasts, endothelial cells, macrophages, dendritic cells, and T cells. The cross talk between tumor cells and surrounding stromal cells is mediated via exosomes. Exosomal cargo, composed of miRNAs and long noncoding RNAs, induces phenotypic alterations in recipient cells, which includes increased cell proliferation, invasiveness, metabolic reprogramming, and/or immunosuppression. Collectively, this contributes to breast cancer progression, metastasis, and drug resistance. (Figure generated with BioRender.com; Toronto, 0N, Canada.) EMT, epithelial-to-mesenchymal transition.

from breast cancer-exosome stimulated HMECs stimulates the growth of breast cancer cell lines. ${ }^{26}$ The first study to examine the miRNA content of exosomes demonstrated an overall enrichment of miRNAs in breast cancer cells compared with exosomes from normal HMECs and exosomes from metastatic breast cancer cells were further enriched with miRNAs compared with nonmetastatic breast cancer cells. It further demonstrated that exosomes perform cell-independent miRNA biogenesis by the processing of pre-miRNAs into mature miRNAs and that the HMEC cell line, MCF10A, on treatment with cancer exosomes had increased cell viability and proliferation, and induced alterations in the transcriptome, including changes to the miR21 and miR-10b targets, phosphatase and tensin homolog and homeobox D10, respectively. In addition, the MCF10A cells were induced to form tumors when co-injected with breast cancer cells or patient serum-derived exosomes into nude mice. ${ }^{14}$ miR-10b is highly expressed in the exosomes from the metastatic triple-negative breast cancer (TNBC) cell line MDA-MB-231. miR-10b can be taken up by HMECs, where it down-regulates its target genes, the homeobox D10 and the transcription factor Kruppel-like factor
4, and promotes invasion. ${ }^{27}$ The transfer of exosomes from TNBC cells to MCF10A cells similarly induces proliferation and a change in miRNA and gene expression programs in the recipient cells, but also transfers chemotherapeutic resistance to docetaxel and doxorubicin compared with untreated MCF10A cells. ${ }^{28}$

The transfer of oncogenic properties via exosomes from one breast cancer cell to another has also been demonstrated. Exosomes from a highly invasive variant of the Hs578T breast cancer cell line increase proliferation, migration, and invasive activity of three recipient breast cancer cell lines. This effect is replicated on the addition of exosomes isolated from serum samples of patients with TNBC as compared with healthy control sera, ${ }^{29}$ although the contents of the exosomes have not been investigated. Several studies have demonstrated the differential expression and exosome transfer of specific miRNAs. miR-1246 was identified as highly abundant in breast cancer exosomes and is highly expressed in the TNBC cell line MDAMB-231. ${ }^{30}$ Exosome transfer of miR-1246 from MDA-MB231 cells to HMECs suppresses the expression of its target gene cyclin-G2 (CCNG2), enhances cell viability, and 
promotes migration and chemotherapy resistance. ${ }^{31}$ Exosome transfer of miR-205 suppresses the expression of the chloride voltage-gated channel 3 (CLCN3), a gene that is up-regulated in Erb-B2 receptor tyrosine kinase 2 (ErbB2)/ human epidermal growth factor receptor 2 (HER2)-overexpressing breast cancer cells and suppresses proliferation of three-dimensional spheroids of MCF10A-ErbB2 overexpressing cells. ${ }^{32}$ Overexpression of miR-1910 in breast cancer cells promotes proliferation, migration, and epithelial-to-mesenchymal programs in vitro and xenograft tumor growth in vivo. Exosome transfer of miR-1910 to MDA-MB-231 and MCF7 breast cancer cells replicates the cancer-promoting effects in vitro and in vivo. In addition, the exosome transfer of miR-1920 promotes autophagyinhibited apoptosis-activated Wnt signaling, and NF- $\kappa$ B signaling through regulation of its target gene myotubularinrelated protein 3 (MTMR3). ${ }^{33}$ Elevated circulating levels of miR-188-5p identified in the serum of breast cancer patients compared with women with breast fibroadenoma or healthy subjects suggest its use as a circulating biomarker. MDAMB-231 cells have lower levels of cellular miR-188-5p compared with MCF7 cells, as indicated by in vitro studies. Functional studies demonstrate that miR-188-5p suppresses proliferation and migration through modulation of its target gene IL-6 cytokine family signal transducer. The exosome fraction of MDA-MB-231 cells has much higher levels of miR-188-5p relative to exosomes from MCF7 cells; however, this was not observed in the exosome fraction of breast cancer patient serum samples. ${ }^{34}$

Few studies have examined the transfer of mRNAs or lncRNAs by exosomes. One study demonstrated that exosomes from breast cancer cells that overexpress the C-X-C motif chemokine receptor 4 , a chemokine receptor that plays a critical role in breast cancer development and progression, contained increased levels of mRNAs related to stem cell differentiation and metastasis. Transfer of exosome from the C-X-C motif chemokine receptor 4 cells promoted proliferation, migration, and invasion in the target cells. Increased levels of stemness- and metastasis-related mRNAs were similarly identified in exosomes from breast cancer patients with poor prognosis. ${ }^{35}$ The IncRNA, metastasis-associated lung adenocarcinoma transcript 1 , is highly up-regulated in multiple cancer types and may function as a molecular scaffold for ribonucleoprotein complexes and as a transcriptional regulator. Metastasis-associated lung adenocarcinoma transcript 1 is elevated in breast cancer patient tissues and serum and is associated with reduced survival. In vitro studies indicate that metastasis-associated lung adenocarcinoma transcript 1 is increased in breast cancer cell lines and exosomes and that metastasis-associated lung adenocarcinoma transcript 1 can be transferred by exosomes and promotes cell proliferation. ${ }^{36}$

Taken together, these studies demonstrate that exosomes from cancer cells can promote oncogenic programs in both cancer and normal cells, suggesting a means by which transformed cancer cells could effectuate other cells within their vicinity to promote cancer development and progression. Although these studies have revealed a significant role for exosomes from fully transformed invasive cells, the exosome-associated intercellular communication at the earliest stages of breast cancer development before invasive progression has not been elucidated.

\section{Promotion of Metastasis}

Exosomes promote metastatic programs, and the role of exosome transfer of RNAs in promoting breast cancer metastasis has recently been reported. The miR-200 family of miRNAs, which is composed of miR-200a, miR-200b, miR-200c, miR-141, and miR-429, is known to modulate breast cancer metastasis through regulation of the epithelialto-mesenchymal -mesenchymal-to-epithelial transition axis. ${ }^{37} \mathrm{miR}-200 \mathrm{c}$ and miR-141 are transcriptionally regulated by the transcription factor forkhead box P3 and lysine acetyltransferase 2B in breast cancer cells. The heterozygous Foxp $3^{\text {sf } /+}$ mouse model, which develops breast tumors and spontaneous lung metastases, indicates a reduction in the miR-200c and miR-141 levels, and an increase in the miR-200b, miR-200a, and miR-429 levels, in the breast tumor cells relative to wild-type mammary epithelial cells. However, increased levels of plasma miR-200c and miR141 were observed during tumor progression and tumor metastasis. Interestingly, circulating levels of these miRNAs are increased in exosomes isolated from the mouse plasma. Similarly, circulating plasma levels of miR-200c and miR141 are elevated in breast cancer patients and are associated with tumor metastasis. ${ }^{38}$ The exosome transfer of miR221 and/or its paralog miR-222 has a described role in both metastasis and chemotherapeutic resistance in breast cancer, ${ }^{39,40}$ of which the latter is further described in the subsequent section. Patients with breast cancer and lymphatic metastasis have high levels of miR-22, and exosome transfer of miR-222 promotes migration and invasion of breast cancer cells in vitro and breast tumor formation in vivo. Regulation of the miR-222 target gene PDZ and LIM domain 2 enhances this effect by blocking its inhibition of the NF- $\kappa$ B pathway. ${ }^{40}$ miR-9 and miR-155 are enriched in MDA-MB-231 metastatic breast cancer exosomes and suppress the expression of their target genes, phosphatase and tensin homolog, and dual-specificity phosphatase 14 , when delivered to recipient nonmetastatic breast cancer cells MCF7. ${ }^{41}$ Exosome miR-4443 promotes breast cancer liver metastasis through the down-regulation of its target gene tissue inhibitor of metalloproteinase 2 and up-regulation of matrix metalloproteinases in the primary tumor and liver of xenograft mice. Interestingly, exosomes from MCF10A cells containing miR-4443 inhibitors directly injected into the primary tumor lead to a reduction in tumor growth and metastases, demonstrating the potential therapeutic efficacy of this exosome-derived miRNA. ${ }^{42}$ Exosomes from a highly brain metastatic cell line with high levels of lncRNA GS16000G8.5 affect the blood-brain barrier integrity and 
promote brain metastasis. Exosomes deficient in this lncRNA failed to promote breast cancer cell infiltration across the blood-brain barrier. ${ }^{43}$ Collectively, these studies demonstrate the potential role of exosome transfer of RNAs in the promotion of breast cancer metastases. However, a comprehensive evaluation of the exosome contents from metastatic breast cancer patients is lacking, and a deeper insight into their combined effect on the development of metastases can provide a better biological understanding and a potential means and rationale for therapeutic intervention targeted at exosome transfer.

\section{Transfer of Drug Resistance}

Drug resistance is a major obstacle in the effective treatment of advanced breast cancer. ${ }^{1}$ Breast cancer can evade chemotherapy, hormonal therapy, and targeted therapy through a multitude of mechanisms. Among them, exosome transfer of RNAs has emerged as an important mechanism by which tumor cells acquire resistance. In 2014, two studies demonstrated that exosomes from docetaxel- and doxorubicin-resistant breast cancer cells can transfer a drugresistance phenotype to recipient drug-sensitive cells. ${ }^{44,45}$ miR-100, miR-222, and miR-30a have been identified as potential mediators of this effect. ${ }^{44}$ Exosome-associated miR-221 (a paralog to miR-222) has been identified as an intermediary of doxorubicin resistance through regulation of its target mRNA phosphoinositide-3-kinase regulatory subunit 1 and subsequent inhibition of the phosphatidylinositol 3-kinase/AKT signaling pathway in target cells. ${ }^{46}$ However, phosphatidylinositol 3-kinase/AKT/mechanistic target of rapamycin pathway activation is an established mechanism of acquired resistance to endocrine and ErbB2/HER2 targeted therapies in breast cancer. Therefore, these findings require additional investigation. ${ }^{47}$ Transfer of doxorubicin resistance is attributed to exosome transfer of miR-155. Elevated exosome levels of miR-155 were identified in exosomes from cancer stem cells and chemoresistant cells, and the exosome-mediated transfer of miR-155 promoted increased migration, epithelial-to-mesenchymal transition, and chemoresistance in chemosensitive cells. ${ }^{48}$ Resistance to platinum therapy in the TNBC cell line, MDA-MB-231, is attributed to the up-regulation of exosomal miR-423-5p. Exosomes from a cisplatin-resistant variant of MDA-MB231 are able to increase cell viability, enhance migration and invasion capabilities, and induce chemoresistance on delivery to cisplatin-sensitive MCF-7 and MDA-MB-231 cells. ${ }^{49}$ Exosome transfer of lncRNAs has also been shown to promote chemoresistance. In one study, the lncRNA H19 was increased in exosomes from doxorubicinresistant breast cancer cells and could transfer this resistance on exosome uptake in doxorubicin-sensitive cells. ${ }^{50}$ Alternatively, reintroduction and exosome delivery of miR-134, which was is highly down-regulated in aggressive breast cancer cells and their exosomes, reduces cancer progressive and enhance sensitivity to cisplatin and anti-Hsp90 drugs through reduction of its target genes STAT5B and Hsp90. ${ }^{51}$

Resistance to antihormonal therapies has also been attributed to the exosome transfer of RNAs. A recent study examined the overall differences in exosome cargo between long-term estrogen-deprived MCF7 cells and found that the long-term estrogen-deprived cells secreted a higher amount of exosomal RNA and proteins relative to wild-type MCF7 cells. Although the content of the RNA fraction was not reported, the most common up-regulated class of proteins included Rab GTPases, which are responsible for the regulation of vesicular transport. ${ }^{52}$ The miR-221/222 paralogs also have a described role in antihormone therapy resistance. Specifically, miR-221/222 from a tamoxifen-resistant variant of estrogen receptor-positive MCF7 breast cancer cells transferred via exosomes to wild-type MCF7 cells released and elevated the cellular levels of miR-221/222 and effectively reduced the expression of their target genes, p27/KIP1 and estrogen receptor $\alpha$, and enhanced tamoxifen resistance in the recipient cells. ${ }^{39}$ Two additional studies have examined the role of the exosome transfer of the lncRNA urothelial carcinoma-associated 1 and the circular RNA UBE2D2 in tamoxifen resistance. ${ }^{53,54}$ These studies found that the exosomes from tamoxifen-resistant cells were enriched with these RNAs and that exosome-mediated transfer was associated with increased tamoxifen resistance in the recipient cells. Specifically, circular RNA UBE2D2 was found to interact with the miR-200a-3p to modulate estrogen receptor $\alpha$ expression and tamoxifen resistance. ${ }^{54}$

Exosome-mediated resistance to targeted therapies, including anti-ErbB2/HER2 monoclonal antibody therapy such as trastuzumab in HER2 ${ }^{+}$breast cancers, and cyclindependent kinase (CDK) 4/6 inhibitors such as palbociclib in estrogen receptor-positive breast cancers, has also been reported in recent years. Two studies examined the exosome transfer of two lncRNAs in resistance to trastuzumab. The IncRNA-small nucleolar RNA host gene 14 was upregulated in trastuzumab-resistant cells and incorporated into exosomes. Exosome transfer of small nucleolar RNA host gene 14 to target cells blocks apoptosis by targeting the B-cell lymphoma 1 (BCL-1)/apoptosis regulator BCL2 associated $\mathrm{X}(\mathrm{BAX})$ signaling pathway. Elevated circulating exosome levels of lncRNA-small nucleolar RNA host gene 14 identified in the serum of patients with trastuzumab resistance relative to those who responded to treatment, suggest that this lncRNA may be used as a biomarker or therapeutic target of resistance mechanisms. ${ }^{55}$ Levels of the lncRNA actin filament-associated protein 1 antisense RNA 1 are elevated in exosomes from trastuzumab resistance breast cancer cells. Exosome actin filament-associated protein 1 antisense RNA 1 is associated with the RNA binding protein AU-binding factor, which promotes the translation of the ErbB2 protein and resistance in recipient cells. ${ }^{56}$ Likewise, another IncRNA, ArfGAP With GTPase Domain, Ankyrin Repeat And PH Domain 2 (AGAP2) antisense RNA 1, is incorporated in exosomes derived from 
trastuzumab resistance breast cancer cells via interaction with the heterogeneous nuclear ribonucleoprotein A2/B1 and promotes resistance on delivery to target cells. ${ }^{57}$ In contrast, the miRNA, miR-567, is down-regulated in trastuzumab-resistant breast cancer cells relative to wildtype cells. Exosome packaging of miR-567 suppresses autophagy and trastuzumab resistance through targeting the autophagy-related $5 \mathrm{mRNA}$, suggesting the development of miR-567 as a therapeutic target for anti-HER2 therapy. ${ }^{58}$ Exosome transfer of another miRNA promotes resistance to CDK4/6 inhibitors. Specifically, the exosome transfer of miR-432-5p from resistance cells to wild-type parental cells promotes resistance to CDK4/6 inhibition through downregulation of the transforming growth factor- $\beta$ (TGF $\beta$ ) pathway, which, in turn, allows for the increased expression of the CDK6. Interestingly, when resistance cells are provided a prolonged drug holiday, the resistance phenotype and levels of exosome miR-432 are reversed, suggesting a potential therapeutic approach to overcome resistance in patients treated with CDK4/6 inhibitors. ${ }^{59}$ Taken together, these studies demonstrate that therapeutic resistance is transferrable via exosomes, and further show that the resistance mechanisms can be reversed, opening up new therapeutic opportunities to address this problem in the effective treatment of breast cancer.

\section{Heterotypic Interactions}

Breast TME is a complex and heterogeneous ecosystem composed of multiple cell types such as cancer-associated fibroblasts, endothelial cells, adipocytes, and immune cells, that participate in different stages of breast cancer initiation and progression. ${ }^{60}$ They interact with tumor cells via exosomes to promote ECM remodeling, inflammation, chemoresistance, and immune suppression, which are critical drivers for tumor progression. ${ }^{14,29,61}$ Exosomes carry diverse functional proteins, lipids, and nucleic acids crucial for cell-cell communication. They transfer their contents to the recipient cells to induce phenotypic changes, thereby generating an environment conducive to tumor progression. In addition to the role of exosomes at the primary tumor site, they also contribute to establishing a niche for metastasizing breast cancer cells at distant organ sites, such as the lungs, brain, and liver. ${ }^{62} \mathrm{~A}$ better understanding of the key signaling pathways modulated by exosomal cargo will help identify novel therapeutic targets to combat this deadly disease. The diverse roles of exosomes in mediating cross talk between tumor and stromal cells at primary and secondary tumor sites are summarized in the following sections.

\section{Cancer-Associated Fibroblasts}

Fibroblasts represent most stromal cells in the TME and express markers, including fibroblast-specific protein-1, fibroblast activation protein, and $\alpha$-smooth muscle actin. They play an important role in regulating inflammation and promoting wound healing and ECM synthesis. In breast cancer, the quiescent fibroblasts are reprogrammed to CAFs, acquiring a highly proliferative state with increased migratory capabilities and dysregulated miRNA expression. ${ }^{63}$ Moreover, CAFs are characterized by aberrant signaling in pathways related to cell adhesion and secretion of factors, such as chemokines, cytokines, and metalloproteinases, which help in cancer progression and metastasis.Exosomemediated delivery of miRNAs promotes the transformation of normal fibroblasts to CAFs in the tumor stroma, thereby contributing to breast cancer aggressiveness. In another study, ${ }^{64}$ exosomes derived from breast cancer patient CAFs shuttled miR-21, miR-143, and miR-378e into T47D breast cancer cells. These CAF-derived exosomes and their miRNAs promote breast cancer cell dedifferentiation, resulting in increased mammosphere formation, and expression of stem cells and EMT markers. Interestingly, these phenotypes are also induced by exosomes derived from normal fibroblasts transfected with miR-378e and miR-143, indicating that exosomal miRNAs can induce stemness and mesenchymal phenotype in breast cancer cells. Furthermore, miR-3613-3p, up-regulated in CAFs and their exosomes, is associated with tumor growth, stress responses, and metastasis in breast cancer via targeting suppressor of cytokine signaling 2 . This highlights the importance of exo-miR-3613-3p as a potential biomarker for breast cancer prognosis. ${ }^{65}$

Differential expression of miRNAs in the exosomes derived from CAFs and normal fibroblasts can be indicative of cancer vulnerabilities that can be therapeutically targeted. One such study identified miR-4516 as an anticancer drug for TNBC. ${ }^{66}$ It showed that miR-4516 was highly downregulated in exosomes derived from CAFs than in normal fibroblast-derived exosomes isolated from an invasive ductal carcinoma patient. miR-4516 overexpression or mimic treatment reduced TNBC cell proliferation via negative regulation of FOS-like 1. In addition, several studies have shown that CAFs and the CAF-derived exosomes can suppress the immune response in breast cancer TME by recruiting M2 macrophages. A recent study showed that exosomes derived from CAFs can induce PD$\mathrm{L} 1$ expression in breast cancer cells, promote $\mathrm{T}$-cell apoptosis, and impair natural killer cell function. This immunosuppressive role of CAFs in breast cancer was also confirmed in vivo and was mechanistically mediated via the miR-92-LATS2-YAP1 axis, which enhances PD-L1 transcriptional activity. ${ }^{67}$ This indicates a novel mechanism contributing to an immunosuppressive TME in breast cancer. Dysregulation of breast cancer cell-derived exosomal IncRNAs also plays an important role in the premetastatic niche formation. Lung fibroblasts malignantly transform by uptake of exosomes derived from MDA-MB-231 cells. ${ }^{68}$ These exosomes have aberrant lncRNA expression and phenotypically affect lung fibroblast proliferation and migration, thereby promoting lung metastasis. 
CAFs also play an important role in metabolic rewiring of the TME, which impacts nutrient dependencies of cancer cells, leading to metabolic reprogramming. Thus, studying the role of exosomes in this bidirectional message transfer from cancer cells to the stromal cells and vice versa in the TME has gained importance. In a recent study, uptake of miR-105 containing breast cancer-derived exosomes by CAFs led to activation of MYC proto-oncogne (MYC) signaling. This allowed the CAFs to exhibit metabolic plasticity, depending on the nutrient availability in the TME, thereby helping to sustain tumor growth. ${ }^{69}$ Collectively, these studies demonstrate that CAFs in the breast TME exhibit heterogeneous phenotypes and exert different functions to promote tumorigenesis and malignant transformation via releasing exosomes, thus making them an attractive therapeutic target for modulating the TME.

\section{Endothelial Cells}

Blood supply plays a critical role in cancer progression as it provides access to oxygen and nutrients in the tumor and a route to eliminate metabolic waste. Angiogenesis, the process of formation of new blood vessels from pre-existing ones, is crucial in tumor progression. Exosomes derived from breast cancer cells can impact the transendothelial migration of cancer cells into the vasculature, but can also destabilize the endothelial cell intercellular junctions to promote breast cancer metastasis to distant sites. ${ }^{70}$ Several studies have demonstrated a reciprocal exchange of factors between endothelial cells and malignant cells in the TME to regulate tumor growth. In one study, ${ }^{71}$ endothelial cell-derived exosomes loaded with miR-503 inhibited tumor growth by directly acting on the tumor cells. The expression of endothelial miR-503 is regulated by neoadjuvant chemotherapy in breast cancer, wherein it inhibits tumor cell proliferation and invasion. This study showed that endothelial exosomes can modulate breast cancer tumor progression on chemotherapy or radiation treatment by directly affecting the tumor cells via antitumor miRNA. In another study, ${ }^{72}$ breast cancer cell-derived exosomes containing miR-21 abrogated the decrease in cell proliferation, migration, and invasion of endothelial progenitor cells, thus resulting in deep vein thrombosis in patients with breast carcinoma.

In early-stage breast cancer, tumor cells disseminate via both hematogenous and lymphatic routes; however, the factors determining the route are largely unknown. Furthermore, patients with TNBC predominantly show more capillary invasion than lymphatic invasion. miR939-containing exosomes derived from invasive breast cancer cell lines such as MDA-MB-231 favor tumor cell migration through the endothelial barrier by targeting vascular endothelial cadherin, a component of adherens junction involved in vessel permeability. ${ }^{73}$ Another study demonstrated that exosome-mediated transfer of cancersecreted miR-105 destroyed tight junctions in endothelial monolayer. ${ }^{74}$ Furthermore, thrombospondin-1 has also been implicated in promoting transendothelial migration of metastatic tumor cells by decreasing the expression of intercellular junction proteins. $^{75}$ Lymphatic vasculature associated with tumors is also a known route of metastatic spread in breast cancer. Extracellular vesicles contribute to this cross talk between lymphatic endothelial cells that line the lymphatic vessels and cancer cells. ETS transcription factor (ELK3), express in lymphatic endothelial cells, regulate exosomal cargo, and promote metastasis by transferring oncogenic exo-miRNAs (miR-503-3p, miR-4269, and miR-30e-3p) to tumor cells. ${ }^{76}$ Taken together, these studies highlight the role of exosomes in promoting the malignant spread of breast cancer cells by disrupting barrier cell integrity and the transfer of oncogenic signals.

Given the important role endothelial cells play in the breast TME, inhibition of angiogenesis is a promising strategy for the development of novel cancer therapeutics. However, drugs that inhibit tumor blood vessel formation by blocking the vascular endothelial growth factor pathway have shown limited clinical success and are associated with adverse events and the development of resistance. ${ }^{77}$ Thus, a better understanding of the interactions mediated via extracellular vesicles between cancer cells and endothelial cells will help identify new therapeutic strategies to improve patient outcomes.

\section{Immune Cells}

Heterogeneous populations of immune cells, like neutrophils, macrophages, lymphocytes, and myeloid-derived suppressor cells present within the TME affect tumor cell proliferation and metastasis. ${ }^{78}$ Exosomes can serve as a source of tumor antigens to stimulate the immune response $^{79}$; however, exosomes derived from tumor cells predominantly support cancer cell survival and progression by mechanisms that help cancer cells escape the immune system. These include modulation of antigen presentation, induction of T-cell apoptosis, and activation of suppressor immune cells. Tumor-derived exosomes can transport proteins like Fas ligand and tumor necrosis factor superfamily member 10 (TRAIL) to immune cells, thereby generating an immunosuppressive microenvironment. ${ }^{80}$ Breast cancer-derived exosomes can also accumulate at metastatic sites, such as the lung, spleen, and bone, where they can reduce T-cell proliferation and induce natural killer cell cytotoxicity. In addition, exosomes also contain multiple miRNAs, such as miR-146a, miR-29a, and miR-21, that affect lymphocyte development and function, leading to reduced immune response. ${ }^{81}$ Exosomal transfer of the lncRNA small nucleolar RNA host gene 16 from breast cancer cells to $\gamma \delta \mathrm{T} 1$ cells (major component of tumorinfiltrating lymphocytes) potentiates the TGF $\beta 1 /$ SMAD5 pathway, leading to immunosuppression in the TME. ${ }^{82}$ Collectively, these studies indicate that targeting cancer 
cell-derived exosomes could be a promising strategy for breast cancer treatment in the future.

Among the different immune cells in TME, the tumorassociated macrophages are critical drivers for generating an inflammatory microenvironment that facilitates breast cancer progression and metastasis. ${ }^{83}$ These macrophages have high plasticity, wherein they can undergo a switch from M1 to M2 phenotype (anti-inflammatory and protumorigenic) based on environmental cues. A recent study ${ }^{84}$ showed that exosomal transfer of miRNAs (miR-100-5p, miR-183-5p, and miR-125b-1-3p) from mouse $4 \mathrm{~T} 1$ breast cancer cells increased macrophage cytokine production of IL-1 $\beta$, IL-6, and tumor necrosis factor- $\alpha(\mathrm{TNF} \alpha)$, thereby contributing to metastatic spread in vivo. In another study, ${ }^{85}$ the transfer of exosomal glycoprotein 130 (gp130) from breast cancer cells to macrophages altered their phenotype via activation of IL-6/gp130-STAT3 signaling pathway, thus making them protumorigenic. Furthermore, exosomal transfer of lncRNA breast cancer-related transcript 1 from cancer cells to macrophages promotes M2 polarization, macrophage migration, and tumor progression. ${ }^{86}$ Uptake of tumorderived miR-375 by tumor-associated macrophages enhance macrophage infiltration and migration into breast cancer cell spheroids, thereby contributing to a protumorigenic environment. ${ }^{87}$ M2 macrophages also secrete exosomes with different miRNAs that can induce phenotypic changes in breast cancer cells. For example, M2 cell-derived exosomes containing miR-223, when taken up by cocultured breast cancer cells, lead to increased invasiveness of the cancer cells, ${ }^{88}$ which is abrogated by adding miR-223 antisense oligonucleotides to cancer cells. Altogether, these studies indicate that strategies to change macrophage polarization from protumoral (M2) to an antitumoral (M1) phenotype will help suppress tumor growth. One such approach includes modification of the miRNA content of cancer cell-derived exosomes that can reprogram macrophages. To this end, miR-130 and miR-33 overexpression in exosomes helped convert tumor-associated macrophages to M1 phenotype and reduced tumor growth. ${ }^{89}$ It is well established that exosomes derived from tumor cells target the immune cells in the TME and can alter their composition and function to help tumors evade the immune response. This opens multiple avenues for the development of novel anticancer therapeutics targeted at the interaction of tumor and immune cells in breast cancer.

\section{Adipocytes}

Adipocytes or fat cells, especially in the mammary adipose tissue, play an active role in the pathologic process of breast cancer progression and metastasis. They are a source of a variety of proinflammatory secretory factors called adipokines that facilitate tumor progression and metastasis. ${ }^{90}$ Current evidence suggests a dynamic cross talk between adipocytes and cancer cells mediated by exosomes. In a symbiotic relationship, cancer cell-derived exosomes induce metabolic reprogramming of fat cells, leading to increased energy expenditure and release of oncometabolities such as lactate, pyruvate, and free fatty acids. ${ }^{10}$ miR-155, derived from breast cancer cells, down-regulates peroxisome proliferator-activated receptor $\gamma$ in adipocytes to regulate their energy metabolism. ${ }^{91}$ This results in increased lipolysis in fat cells and loss of muscles, leading to cancer-associated cachexia. In turn, adipocytes promote breast tumor invasiveness by inducing the epithelial-to-mesenchymal program in cancer cells. ${ }^{92}$ A study highlighting the importance of preadipocyte-derived exosomes in modulating breast cancer cell behavior showed that exosomes derived from preadipocytes activate SRY-Box Transcription Factor 9 (SOX9) in ductal carcinoma in situ cells via miR-140. ${ }^{93}$ Activation of miR-140/SOX9 pathway promotes mammosphere formation, stemness, and migration of ductal carcinoma in situ cells in vitro. Moreover, adipocytes also play an important role in conferring drug resistance to the tumor cells by modulation of the cell death pathways. Exosomes derived from adipose tissue mesenchymal stem cells contain miR-1236, which down-regulates Solute Carrier Family 9 Member A1 (SLC9A1) and the Wnt/ $\beta$-catenin pathway. ${ }^{94}$ This sensitizes the breast cancer cells to cisplatin treatment. Another study ${ }^{95}$ showed increased resistance to doxorubicin in breast cancer cells in the presence of adipocytes. Factors secreted by adipocytes influence the redistribution of doxorubicin in the tumor cells from the nucleus to cytoplasmic vesicles, thereby increasing efflux of the drug into extracellular media via exosomes that contribute to the resistant phenotype. Overall, cancerassociated adipocytes facilitate tumor progression and metastasis by not only releasing growth factors and cytokines but also by playing a crucial role in metabolic reprogramming of cancer cells, facilitating invasion by releasing matrix-degrading enzymes and helping to recruit and activate inflammatory cells in the breast TME. Given their pivotal role in the breast cancer TME, it is imperative to better understand the reciprocal interactions between cancer cells and adipocytes for the development of better TME-modulating therapies and to overcome therapeutic resistance.

\section{Current Strategies for Pharmacologic Intervention of Exosome-mediated Intercellular Communication}

Exosomes are known to facilitate cell-to-cell communication, and a better understanding of their biological functions in breast cancer progression and metastasis has provided promising avenues that can be exploited therapeutically. Exosomes have been used to successfully deliver drugs and therapeutic miRNAs because of their natural delivery capabilities. They have transmembrane and membrane anchoring proteins that help in endocytosis and content transfer. As a result, they are not 
associated with poor bioavailability and/or non-specific cytotoxicity and immunogenicity. ${ }^{96}$ In a recent study,${ }^{97}$ exosomes derived from macrophages were engineered to target mesenchymal-epithelial transition factor, which is overexpressed in TNBC. In addition, these modifications also enhanced cellular uptake and cytotoxic effects of doxorubicin. ${ }^{97}$ Multiple small-molecule inhibitors have been developed to either reduce the release of exosomes from cancer cells or decrease their uptake by target cells [eg, ras-related protein (Rab27A) inhibitors, neutral sphingomyelinase inhibitors, proton pump inhibitors, and calcium channel blockers]. ${ }^{98,99}$ Some of the compounds (eg, tipifarnib and ketoconazole) selectively affect the exosome secretion from tumor cells and spare the normal cells. Exosomes in the TME have a dual effect on the immune cells. They can not only act as vehicles for the delivery of tumor antigens but are also able to blunt the immune response to help tumor cells evade the immune system. An alternative approach being tested in a clinical trial is to use a Hemopurifier $^{\mathrm{TM}}$ to deplete immunosuppressive exosomes from the blood before pembrolizumab (immunotherapeutic) treatment in patients with metastatic head and neck squamous cell carcinoma (ClinicalTrials.gov, identifier: NCT04453046). Furthermore, the efficacy of a cancer vaccine containing tumor antigen-loaded exosomes derived from dendritic cells is also under evaluation in patients with unresectable non-small-cell lung cancer (ClinicalTrials.gov, identifier: NCT01159288). Another phase 1 clinical trial (ClinicalTrials. gov, identifier: NCT03608631) in metastatic pancreatic cancer patients (with KrasG12D mutation) is underway to assess maximum tolerated dose and disease control by using exosomes loaded with KrasG12D siRNA, derived from mesenchymal stromal cells. The role of cancer cell-derived exosomes in breast cancer clinical studies has not been well established, although there is a lot of preclinical evidence that highlights their functional relevance in TME. In a recently initiated breast cancer clinical trial (ClinicalTrials.gov, identifier: NCT04288141), the expression of a HER2-HER3 dimer will be assessed in exosomes isolated from blood of patients receiving HER2 targeted therapies (trastuzumab and pertuzumab). Herein, assessment of patient-derived exosomes will help identify individuals responsive to anti-HER2 treatments and predict the development of drug resistance.

Overall, the design of tumor cell-specific engineered exosomes and the development of exosome-specific inhibitors may play an important role in the future clinical management of breast cancer by modulating different aspects of the TME. To ensure clinical success, the pharmacologic agents need to be more selective for exosomal release/uptake in tumor/stromal cells and without systemic adverse effects. Also, the agents may be used in combination with other agents to improve therapeutic efficacy. For example, inhibition of tumor exosome release or depletion of HER2 ${ }^{+}$circulating exosomes with trastuzumab therapy in HER2 ${ }^{+}$breast cancer patients. Furthermore, exosomes could be engineered to deliver targeted drugs or tumorsuppressive proteins as a precision medicine approach to cancer treatment. Future studies are required to better understand the dynamic and kinetic changes in exosomes in the TME following chemotherapy/radiotherapy in breast cancer patients to develop more effective combination treatment strategies.

\section{References}

1. Harbeck N, Penault-Llorca F, Cortes J, Gnant M, Houssami N, Poortmans P, Ruddy K, Tsang J, Cardoso F: Breast cancer. Nat Rev Dis Primers 2019, 5:66

2. Aubele M, Mattis A, Zitzelsberger H, Walch A, Kremer M, Welzl G, Hofler H, Werner M: Extensive ductal carcinoma in situ with small foci of invasive ductal carcinoma: evidence of genetic resemblance by CGH. Int J Cancer 2000, 85:82-86

3. Kim SY, Jung SH, Kim MS, Baek IP, Lee SH, Kim TM, Chung YJ, Lee SH: Genomic differences between pure ductal carcinoma in situ and synchronous ductal carcinoma in situ with invasive breast cancer. Oncotarget 2015, 6:7597-7607

4. Kroigard AB, Larsen MJ, Laenkholm AV, Knoop AS, Jensen JD, Bak M, Mollenhauer J, Kruse TA, Thomassen M: Clonal expansion and linear genome evolution through breast cancer progression from pre-invasive stages to asynchronous metastasis. Oncotarget 2015, 6 : $5634-5649$

5. Lee S, Stewart S, Nagtegaal I, Luo J, Wu Y, Colditz G, Medina D, Allred DC: Differentially expressed genes regulating the progression of ductal carcinoma in situ to invasive breast cancer. Cancer Res 2012, 72:4574-4586

6. Muggerud AA, Hallett M, Johnsen H, Kleivi K, Zhou W, Tahmasebpoor S, Amini RM, Botling J, Borresen-Dale AL, Sorlie T, Warnberg F: Molecular diversity in ductal carcinoma in situ (DCIS) and early invasive breast cancer. Mol Oncol 2010, 4: 357-368

7. Quail DF, Joyce JA: Microenvironmental regulation of tumor progression and metastasis. Nat Med 2013, 19:1423-1437

8. Barcellos-Hoff MH, Lyden D, Wang TC: The evolution of the cancer niche during multistage carcinogenesis. Nat Rev Cancer 2013, 13: $511-518$

9. Dias AS, Almeida CR, Helguero LA, Duarte IF: Metabolic crosstalk in the breast cancer microenvironment. Eur J Cancer 2019, 121: $154-171$

10. Dirat B, Bochet L, Dabek M, Daviaud D, Dauvillier S, Majed B, Wang YY, Meulle A, Salles B, Le Gonidec S, Garrido I, Escourrou G, Valet P, Muller C: Cancer-associated adipocytes exhibit an activated phenotype and contribute to breast cancer invasion. Cancer Res 2011, 71:2455-2465

11. Hannafon BN, Ding WQ: Intercellular communication by exosomederived microRNAs in cancer. Int J Mol Sci 2013, 14:14240-14269

12. Ruivo CF, Adem B, Silva M, Melo SA: The biology of cancer exosomes: insights and new perspectives. Cancer Res 2017, 77: 6480-6488

13. Antonyak MA, Li B, Boroughs LK, Johnson JL, Druso JE, Bryant KL, Holowka DA, Cerione RA: Cancer cell-derived microvesicles induce transformation by transferring tissue transglutaminase and fibronectin to recipient cells. Proc Natl Acad Sci U S A 2011, 108:4852-4857

14. Melo SA, Sugimoto H, O'Connell JT, Kato N, Villanueva A Vidal A, Qiu L, Vitkin E, Perelman LT, Melo CA, Lucci A, Ivan C, Calin GA, Kalluri R: Cancer exosomes perform cell-independent microRNA biogenesis and promote tumorigenesis. Cancer Cell 2014, 26:707-721

15. Allenson K, Castillo J, San Lucas FA, Scelo G, Kim DU, Bernard V, Davis G, Kumar T, Katz M, Overman MJ, Foretova L, Fabianova E, Holcatova I, Janout V, Meric-Bernstam F, Gascoyne P, Wistuba I, Varadhachary G, Brennan P, Hanash S, Li D, Maitra A, Alvarez H: 
High prevalence of mutant KRAS in circulating exosome-derived DNA from early-stage pancreatic cancer patients. Ann Oncol 2017, 28:741-747

16. Keerthikumar S, Chisanga D, Ariyaratne D, Al Saffar H, Anand S, Zhao K, Samuel M, Pathan M, Jois M, Chilamkurti N, Gangoda L, Mathivanan S: ExoCarta: a web-based compendium of exosomal cargo. J Mol Biol 2016, 428:688-692

17. Murillo OD, Thistlethwaite W, Rozowsky J, Subramanian SL, Lucero R, Shah N, et al: exRNA atlas analysis reveals distinct extracellular RNA cargo types and their carriers present across human biofluids. Cell 2019, 177:463-477.e15

18. Skog J, Wurdinger T, van Rijn S, Meijer DH, Gainche L, SenaEsteves M, Curry WT Jr, Carter BS, Krichevsky AM, Breakefield XO: Glioblastoma microvesicles transport RNA and proteins that promote tumour growth and provide diagnostic biomarkers. Nat Cell Biol 2008, 10:1470-1476

19. Valadi H, Ekstrom K, Bossios A, Sjostrand M, Lee JJ, Lotvall JO: Exosome-mediated transfer of mRNAs and microRNAs is a novel mechanism of genetic exchange between cells. Nat Cell Biol 2007, 9:654-659

20. Pegtel DM, Cosmopoulos K, Thorley-Lawson DA, van Eijndhoven MA, Hopmans ES, Lindenberg JL, de Gruijl TD, Wurdinger T, Middeldorp JM: Functional delivery of viral miRNAs via exosomes. Proc Natl Acad Sci U S A 2010, 107:6328-6333

21. McAndrews KM, Kalluri R: Mechanisms associated with biogenesis of exosomes in cancer. Mol Cancer 2019, 18:52

22. Kalluri R, LeBleu VS: The biology, function, and biomedical applications of exosomes. Science 2020, 367:eaau6977

23. Sun Y, You S, Tu H, Spillman DR Jr, Chaney EJ, Marjanovic M, Li J, Barkalifa R, Wang J, Higham AM, Luckey NN, Cradock KA, George Liu Z, Boppart SA: Intraoperative visualization of the tumor microenvironment and quantification of extracellular vesicles by label-free nonlinear imaging. Sci Adv 2018, 4:eaau5603

24. Tu H, Liu Y, Marjanovic M, Chaney EJ, You S, Zhao Y, Boppart SA: Concurrence of extracellular vesicle enrichment and metabolic switch visualized label-free in the tumor microenvironment. Sci Adv 2017, 3:e1600675

25. You S, Barkalifa R, Chaney EJ, Tu H, Park J, Sorrells JE, Sun Y, Liu YZ, Yang L, Chen DZ, Marjanovic M, Sinha S, Boppart SA: Label-free visualization and characterization of extracellular vesicles in breast cancer. Proc Natl Acad Sci U S A 2019, 116:24012-24018

26. Dutta S, Warshall C, Bandyopadhyay C, Dutta D, Chandran B: Interactions between exosomes from breast cancer cells and primary mammary epithelial cells leads to generation of reactive oxygen species which induce DNA damage response, stabilization of p53 and autophagy in epithelial cells. PLoS One 2014, 9:e97580

27. Singh R, Pochampally R, Watabe K, Lu Z, Mo YY: Exosomemediated transfer of miR-10b promotes cell invasion in breast cancer. Mol Cancer 2014, 13:256

28. Ozawa PMM, Alkhilaiwi F, Cavalli IJ, Malheiros D, de Souza Fonseca Ribeiro EM, Cavalli LR: Extracellular vesicles from triplenegative breast cancer cells promote proliferation and drug resistance in non-tumorigenic breast cells. Breast Cancer Res Treat 2018, $172: 713-723$

29. O'Brien K, Rani S, Corcoran C, Wallace R, Hughes L, Friel AM, McDonnell S, Crown J, Radomski MW, O'Driscoll L: Exosomes from triple-negative breast cancer cells can transfer phenotypic traits representing their cells of origin to secondary cells. Eur J Cancer 2013, 49:1845-1859

30. Hannafon BN, Trigoso YD, Calloway CL, Zhao YD, Lum DH, Welm AL, Zhao ZJ, Blick KE, Dooley WC, Ding WQ: Plasma exosome microRNAs are indicative of breast cancer. Breast Cancer Res 2016, 18:90

31. Li XJ, Ren ZJ, Tang JH, Yu Q: Exosomal microRNA miR-1246 promotes cell proliferation, invasion and drug resistance by targeting CCNG2 in breast cancer. Cell Physiol Biochem 2017, 44:1741-1748

32. Takeno T, Hasegawa T, Hasegawa H, Ueno Y, Hamataka R, Nakajima A, Okubo J, Sato K, Sakamaki T: MicroRNA-205-5p inhibits three-dimensional spheroid proliferation of ErbB2overexpressing breast epithelial cells through direct targeting of CLCN3. Peer J 2019, 7:e7799

33. Wang B, Mao JH, Wang BY, Wang LX, Wen HY, Xu LJ, Fu JX, Yang H: Exosomal miR-1910-3p promotes proliferation, metastasis, and autophagy of breast cancer cells by targeting MTMR3 and activating the NF- $\mathrm{B}$ signaling pathway. Cancer Lett 2020, 489:87-99

34. Wang M, Zhang H, Yang F, Qiu R, Zhao X, Gong Z, Yu W, Zhou B, Shen B, Zhu W: miR-188-5p suppresses cellular proliferation and migration via IL6ST: a potential noninvasive diagnostic biomarker for breast cancer. J Cell Physiol 2020, 235:4890-4901

35. Rodríguez M, Silva J, Herrera A, Herrera M, Peña C, Martín P, GilCalderón B, Larriba MJ, Coronado MJ, Soldevilla B, Turrión VS, Provencio M, Sánchez A, Bonilla F, García-Barberán V: Exosomes enriched in stemness/metastatic-related mRNAS promote oncogenic potential in breast cancer. Oncotarget 2015, 6:40575-40587

36. Zhang P, Zhou H, Lu K, Lu Y, Wang Y, Feng T: Exosome-mediated delivery of MALAT1 induces cell proliferation in breast cancer. Onco Targets Ther 2018, 11:291-299

37. Nieto MA, Huang RY, Jackson RA, Thiery JP: Emt: 2016. Cell 2016, $166: 21-45$

38. Zhang G, Zhang W, Li B, Stringer-Reasor E, Chu C, Sun L, Bae S, Chen D, Wei S, Jiao K, Yang WH, Cui R, Liu R, Wang L: MicroRNA-200c and microRNA-141 are regulated by a FOXP3-KAT2B axis and associated with tumor metastasis in breast cancer. Breast Cancer Res 2017, 19:73

39. Wei Y, Lai X, Yu S, Chen S, Ma Y, Zhang Y, Li H, Zhu X, Yao L, Zhang J: Exosomal miR-221/222 enhances tamoxifen resistance in recipient ER-positive breast cancer cells. Breast Cancer Res Treat 2014, 147:423-431

40. Ding J, Xu Z, Zhang Y, Tan C, Hu W, Wang M, Xu Y, Tang J: Exosome-mediated miR-222 transferring: an insight into NF- $\mathrm{KB}-$ mediated breast cancer metastasis. Exp Cell Res 2018, 369:129-138

41. Kia V, Paryan M, Mortazavi Y, Biglari A, Mohammadi-Yeganeh S: Evaluation of exosomal miR-9 and miR-155 targeting PTEN and DUSP14 in highly metastatic breast cancer and their effect on low metastatic cells. J Cell Biochem 2019, 120:5666-5676

42. Wang J, Zhang Q, Wang D, Yang S, Zhou S, Xu H, Zhang H, Zhong S, Feng J: Microenvironment-induced TIMP2 loss by cancersecreted exosomal miR-4443 promotes liver metastasis of breast cancer. J Cell Physiol 2020, 235:5722-5735

43. Lu Y, Chen L, Li L, Cao Y: Exosomes derived from brain metastatic breast cancer cells destroy the blood-brain barrier by carrying lncRNA GS1-600G8.5. Biomed Res Int 2020, 2020:7461727

44. Chen WX, Liu XM, Lv MM, Chen L, Zhao JH, Zhong SL, Ji MH, Hu Q, Luo Z, Wu JZ, Tang JH: Exosomes from drug-resistant breast cancer cells transmit chemoresistance by a horizontal transfer of microRNAs. PLoS One 2014, 9:e95240

45. Chen WX, Cai YQ, Lv MM, Chen L, Zhong SL, Ma TF, Zhao JH, Tang JH: Exosomes from docetaxel-resistant breast cancer cells alter chemosensitivity by delivering microRNAs. Tumour Biol 2014, 35: 9649-9659

46. Pan X, Hong X, Lai J, Cheng L, Cheng Y, Yao M, Wang R, Hu N: Exosomal microRNA-221-3p confers adriamycin resistance in breast cancer cells by targeting PIK3R1. Front Oncol 2020, 10:441

47. Guerrero-Zotano A, Mayer IA, Arteaga CL: PI3K/AKT/mTOR: role in breast cancer progression, drug resistance, and treatment. Cancer Metastasis Rev 2016, 35:515-524

48. Santos JC, Lima NDS, Sarian LO, Matheu A, Ribeiro ML, Derchain SFM: Exosome-mediated breast cancer chemoresistance via miR-155 transfer. Sci Rep 2018, 8:829

49. Wang B, Zhang Y, Ye M, Wu J, Ma L, Chen H: Cisplatin-resistant MDA-MB-231 cell-derived exosomes increase the resistance of recipient cells in an exosomal miR-423-5p-dependent manner. Curr Drug Metab 2019, 20:804-814

50. Wang X, Pei X, Guo G, Qian X, Dou D, Zhang Z, Xu X, Duan X: Exosome-mediated transfer of long noncoding RNA H19 induces 
doxorubicin resistance in breast cancer. J Cell Physiol 2020, 235: 6896-6904

51. O'Brien K, Lowry MC, Corcoran C, Martinez VG, Daly M, Rani S, Gallagher WM, Radomski MW, MacLeod RA, O'Driscoll L: miR134 in extracellular vesicles reduces triple-negative breast cancer aggression and increases drug sensitivity. Oncotarget 2015, 6: 32774-32789

52. Augimeri G, La Camera G, Gelsomino L, Giordano C, Panza S, Sisci D, Morelli C, Győrffy B, Bonofiglio D, Andò S, Barone I, Catalano S: Evidence for enhanced exosome production in aromatase inhibitor-resistant breast cancer cells. Int J Mol Sci 2020, 21:5841

53. Xu CG, Yang MF, Ren YQ, Wu CH, Wang LQ: Exosomes mediated transfer of lncRNA UCA1 results in increased tamoxifen resistance in breast cancer cells. Eur Rev Med Pharmacol Sci 2016, 20:4362-4368

54. Hu K, Liu X, Li Y, Li Q, Xu Y, Zeng W, Zhong G, Yu C: Exosomes mediated transfer of circ_UBE2D2 enhances the resistance of breast cancer to tamoxifen by binding to miR-200a-3p. Med Sci Monit 2020, 26:e922253

55. Dong H, Wang W, Chen R, Zhang Y, Zou K, Ye M, He X, Zhang F, Han J: Exosome-mediated transfer of IncRNA-SNHG14 promotes trastuzumab chemoresistance in breast cancer. Int J Oncol 2018, 53: $1013-1026$

56. Han M, Gu Y, Lu P, Li J, Cao H, Li X, Qian X, Yu C, Yang Y, Yang $X$, Han N, Dou D, Hu J, Dong H: Exosome-mediated lncRNA AFAP1-AS1 promotes trastuzumab resistance through binding with AUF1 and activating ERBB2 translation. Mol Cancer 2020, 19:26

57. Zheng Z, Chen M, Xing P, Yan X, Xie B: Increased expression of exosomal AGAP2-AS1 (AGAP2 antisense RNA 1) in breast cancer cells inhibits trastuzumab-induced cell cytotoxicity. Med Sci Monit 2019, 25:2211-2220

58. Han M, Hu J, Lu P, Cao H, Yu C, Li X, Qian X, Yang X, Yang Y, Han N, Dou D, Zhang F, Ye M, Yang C, Gu Y, Dong H: Exosometransmitted miR-567 reverses trastuzumab resistance by inhibiting ATG5 in breast cancer. Cell Death Dis 2020, 11:43

59. Cornell L, Wander SA, Visal T, Wagle N, Shapiro GI: MicroRNAmediated suppression of the TGF- $\beta$ pathway confers transmissible and reversible CDK4/6 inhibitor resistance. Cell Rep 2019, 26: 2667-2680.e7

60. Tabassum DP, Polyak K: Tumorigenesis: it takes a village. Nat Rev Cancer 2015, 15:473-483

61. Menck K, Scharf C, Bleckmann A, Dyck L, Rost U, Wenzel D, Dhople VM, Siam L, Pukrop T, Binder C, Klemm F: Tumor-derived microvesicles mediate human breast cancer invasion through differentially glycosylated EMMPRIN. J Mol Cell Biol 2015, 7:143-153

62. Fidler IJ: The pathogenesis of cancer metastasis: the "seed and soil" hypothesis revisited. Nat Rev Cancer 2003, 3:453-458

63. Park SY, Kim HM, Koo JS: Differential expression of cancerassociated fibroblast-related proteins according to molecular subtype and stromal histology in breast cancer. Breast Cancer Res Treat 2015, 149:727-741

64. Donnarumma E, Fiore D, Nappa M, Roscigno G, Adamo A, Iaboni M, Russo V, Affinito A, Puoti I, Quintavalle C, Rienzo A, Piscuoglio S, Thomas R, Condorelli G: Cancer-associated fibroblasts release exosomal microRNAs that dictate an aggressive phenotype in breast cancer. Oncotarget 2017, 8:19592-19608

65. Liu Y, Yang Y, Du J, Lin D, Li F: MiR-3613-3p from carcinomaassociated fibroblasts exosomes promoted breast cancer cell proliferation and metastasis by regulating SOCS2 expression. IUBMB Life 2020, 72:1705-1714

66. Kim JE, Kim BG, Jang Y, Kang S, Lee JH, Cho NH: The stromal loss of miR-4516 promotes the FOSL1-dependent proliferation and malignancy of triple negative breast cancer. Cancer Lett 2020, 469: 256-265

67. Dou D, Ren X, Han M, Xu X, Ge X, Gu Y, Wang X: Cancerassociated fibroblasts-derived exosomes suppress immune cell function in breast cancer via the miR-92/PD-L1 pathway. Front Immunol 2020, 11:2026

68. Feng T, Zhang P, Sun Y, Wang Y, Tong J, Dai H, Hua Z: High throughput sequencing identifies breast cancer-secreted exosomal LncRNAs initiating pulmonary pre-metastatic niche formation. Gene 2019, 710:258-264

69. Yan W, Wu X, Zhou W, Fong MY, Cao M, Liu J, Liu X, Chen CH, Fadare O, Pizzo DP, Wu J, Liu L, Liu X, Chin AR, Ren X, Chen Y, Locasale JW, Wang SE: Cancer-cell-secreted exosomal miR-105 promotes tumour growth through the MYC-dependent metabolic reprogramming of stromal cells. Nat Cell Biol 2018, 20:597-609

70. Kikuchi K, McNamara KM, Miki Y, Iwabuchi E, Kanai A, Miyashita M, Ishida T, Sasano H: S100P and ezrin promote transendothelial migration of triple negative breast cancer cells. Cell Oncol (Dordr) 2019, 42:67-80

71. Bovy N, Blomme B, Frères P, Dederen S, Nivelles O, Lion M, Carnet O, Martial JA, Noël A, Thiry M, Jérusalem G, Josse C, Bours V, Tabruyn SP, Struman I: Endothelial exosomes contribute to the antitumor response during breast cancer neoadjuvant chemotherapy via microRNA transfer. Oncotarget 2015, 6: 10253-10266

72. Wang W, Yuan X, Xu A, Zhu X, Zhan Y, Wang S, Liu M: Human cancer cells suppress behaviors of endothelial progenitor cells through miR-21 targeting IL6R. Microvasc Res 2018, 120: $21-28$

73. Di Modica M, Regondi V, Sandri M, Iorio MV, Zanetti A, Tagliabue E, Casalini P, Triulzi T: Breast cancer-secreted miR-939 downregulates VE-cadherin and destroys the barrier function of endothelial monolayers. Cancer Lett 2017, 384:94-100

74. Zhou W, Fong MY, Min Y, Somlo G, Liu L, Palomares MR, Yu Y, Chow A, O'Connor ST, Chin AR, Yen Y, Wang Y, Marcusson EG, Chu P, Wu J, Wu X, Li AX, Li Z, Gao H, Ren X, Boldin MP, Lin PC, Wang SE: Cancer-secreted miR-105 destroys vascular endothelial barriers to promote metastasis. Cancer Cell 2014, 25:501-515

75. Cen J, Feng L, Ke H, Bao L, Li LZ, Tanaka Y, Weng J, Su L: Exosomal thrombospondin-1 disrupts the integrity of endothelial intercellular junctions to facilitate breast cancer cell metastasis. Cancers (Basel) 2019, 11:1946

76. Kim KS, Park JI, Oh N, Cho HJ, Park JH, Park KS: ELK3 expressed in lymphatic endothelial cells promotes breast cancer progression and metastasis through exosomal miRNAs. Sci Rep 2019, 9:8418

77. Carmeliet P: Angiogenesis in life, disease and medicine. Nature 2005, 438:932-936

78. Barriga V, Kuol N, Nurgali K, Apostolopoulos V: The complex interaction between the tumor micro-environment and immune checkpoints in breast cancer. Cancers (Basel) 2019, 11:1205

79. Czernek L, Duchler M: Functions of cancer-derived extracellular vesicles in immunosuppression. Arch Immunol Ther Exp (Warsz) 2017, 65:311-323

80. Wen SW, Sceneay J, Lima LG, Wong CS, Becker M, Krumeich S, Lobb RJ, Castillo V, Wong KN, Ellis S, Parker BS, Möller A: The biodistribution and immune suppressive effects of breast cancerderived exosomes. Cancer Res 2016, 76:6816-6827

81. Cereghetti DM, Lee PP: Tumor-derived exosomes contain microRNAs with immunological function: implications for a novel immunosuppression mechanism. Microrna 2014, 2:194-204

82. Ni C, Fang QQ, Chen WZ, Jiang JX, Jiang Z, Ye J, Zhang T, Yang L, Meng FB, Xia WJ, Zhong M, Huang J: Breast cancer-derived exo-

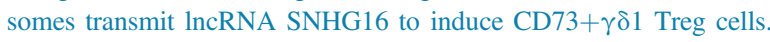
Signal Transduct Target Ther 2020, 5:41

83. Mantovani A, Marchesi F, Malesci A, Laghi L, Allavena P: Tumourassociated macrophages as treatment targets in oncology. Nat Rev Clin Oncol 2017, 14:399-416

84. Guo J, Duan Z, Zhang C, Wang W, He H, Liu Y, Wu P, Wang S, Song M, Chen H, Chen C, Si Q, Xiang R, Luo Y: Mouse 4T1 breast cancer cell-derived exosomes induce proinflammatory cytokine 
production in macrophages via miR-183. J Immunol 2020, 205: $2916-2925$

85. Ham S, Lima LG, Chai EPZ, Muller A, Lobb RJ, Krumeich S, Wen SW, Wiegmans AP, Möller A: Breast cancer-derived exosomes alter macrophage polarization via gp130/STAT3 signaling. Front Immunol 2018, 9:871

86. Liang Y, Song X, Li Y, Chen B, Zhao W, Wang L, Zhang H, Liu Y, Han D, Zhang N, Ma T, Wang Y, Ye F, Luo D, Li X, Yang Q: LncRNA BCRT1 promotes breast cancer progression by targeting miR-1303/PTBP3 axis. Mol Cancer 2020, 19:85

87. Frank AC, Ebersberger S, Fink AF, Lampe S, Weigert A, Schmid T, Ebersberger I, Syed SN, Brüne B: Apoptotic tumor cell-derived microRNA-375 uses CD36 to alter the tumor-associated macrophage phenotype. Nat Commun 2019, 10:1135

88. Yang M, Chen J, Su F, Yu B, Su F, Lin L, Liu Y, Huang JD, Song E: Microvesicles secreted by macrophages shuttle invasion-potentiating microRNAs into breast cancer cells. Mol Cancer 2011, 10:117

89. Moradi-Chaleshtori M, Bandehpour M, Soudi S, MohammadiYeganeh S, Hashemi SM: Correction to: in vitro and in vivo evaluation of anti-tumoral effect of M1 phenotype induction in macrophages by miR-130 and miR-33 containing exosomes. Cancer Immunol Immunother 2021, 70:1323-1339

90. Robado de Lope L, Alcibar OL, Amor Lopez A, HerguetaRedondo M, Peinado H: Tumour-adipose tissue crosstalk: fuelling tumour metastasis by extracellular vesicles. Philos Trans R Soc Lond B Biol Sci 2018, 373:20160485

91. Wu Q, Sun S, Li Z, Yang Q, Li B, Zhu S, Wang L, Wu J, Yuan J, Wang C, Li J, Sun S: Breast cancer-released exosomes trigger cancer-associated cachexia to promote tumor progression. Adipocyte 2019, 8:31-45
92. Wu Q, Sun S, Li Z, Yang Q, Li B, Zhu S, Wang L, Wu J, Yuan J, Yang C, Li J, Sun S: Tumour-originated exosomal miR-155 triggers cancer-associated cachexia to promote tumour progression. Mol Cancer 2018, 17:155

93. Gernapudi R, Yao Y, Zhang Y, Wolfson B, Roy S, Duru N, Eades G, Yang P, Zhou Q: Targeting exosomes from preadipocytes inhibits preadipocyte to cancer stem cell signaling in early-stage breast cancer. Breast Cancer Res Treat 2015, 150:685-695

94. Jia Z, Zhu H, Sun H, Hua Y, Zhang G, Jiang J, Wang X: Adipose mesenchymal stem cell-derived exosomal microRNA-1236 reduces resistance of breast cancer cells to cisplatin by suppressing SLC9A1 and the Wnt/ $\beta$-catenin signaling. Cancer Manag Res 2020, 12: 8733-8744

95. Lehuede C, Li X, Dauvillier S, Vaysse C, Franchet C, Clement E, Esteve D, Longue M, Chaltiel L, Le Gonidec S, Lazar I, Geneste A, Dumontet C, Valet P, Nieto L, Fallone F, Muller C: Adipocytes promote breast cancer resistance to chemotherapy, a process amplified by obesity: role of the major vault protein (MVP). Breast Cancer Res 2019, 21:7

96. Gilligan KE, Dwyer RM: Engineering exosomes for cancer therapy. Int J Mol Sci 2017, 18:1122

97. Li S, Wu Y, Ding F, Yang J, Li J, Gao X, Zhang C, Feng J: Engineering macrophage-derived exosomes for targeted chemotherapy of triple-negative breast cancer. Nanoscale 2020, 12:10854-10862

98. Hessvik NP, Llorente A: Current knowledge on exosome biogenesis and release. Cell Mol Life Sci 2018, 75:193-208

99. Zhang H, Lu J, Liu J, Zhang G, Lu A: Advances in the discovery of exosome inhibitors in cancer. J Enzyme Inhib Med Chem 2020, 35: $1322-1330$ 